\title{
TRIM38 expression associates with survival in triple negative breast cancer.
}

Shahan Mamoor, $\mathrm{MS}^{1}$

${ }^{1}$ shahanmamoor@gmail.com

East Islip, NY USA

We mined published microarray data (1) to understand the most significant gene expression differences in the tumors of triple negative breast cancer patients based on survival at time of analysis: dead or alive. The E3 ubiquitin ligase TRIM38 emerged as among the most significant differences, transcriptome-wide, when comparing the primary tumors of triple negative breast cancer patients dead or alive. Importantly, TRIM38 expression was significantly correlated with overall survival in basal subtype breast cancer, a molecular subtype sharing significant overlap with triple negative breast cancer. TRIM38 may be of relevance as a biomarker or as a molecule of interest in understanding the etiology or progression of triple negative breast cancer.

Keywords: triple negative breast cancer, TNBC, systems biology of breast cancer, targeted therapeutics in breast cancer, TRIM38, tripartite motif containing 38. 
Triple negative breast cancer is defined by lack of expression of the receptors for the hormones progesterone and estrogen (PR negative and ER negative), as well as for the human epidermal growth factor receptor 2 (HER2 negative) $(2,3)$. Though not completely identical, triple negative breast cancer shares significant overlap with the basal or basal-like molecular subtype of human breast cancer (3). TNBC is more frequently diagnosed in women of African descent (black women) for reasons not understood, and their disease course is similarly more aggressive than in white women with triple negative breast cancer $(2,3)$. To understand the transcriptional biology of triple negative breast cancer in an unbiased fashion and at the systems level, we mined published microarray data paired with patient survival data (1), performing comparative transcriptome analysis based on survival at time of data collection: dead or alive. We present here a finding from the preliminary results of this analysis.

\section{Methods}

We used dataset GSE142102 (1) for this differential gene expression analysis in conjunction with GEO2R. GSE142102 was generated using Affymetrix Human Gene 2.1 ST Array technology; in this analysis, we used data from $n=87$ tumors from TNBC patients that were dead at time of analysis and $n=123$ tumors from TNBC patients that were alive at time of analysis. The Benjamini and Hochberg method of $p$-value adjustment was used for ranking of differential expression but raw $p$-values were used to assess statistical significance of global differential expression. Log-transformation of data was auto-detected, and the NCBI generated category of platform annotation was used. A statistical test was performed to evaluate whether TRIM38 expression was significantly different between the primary tumors of TNBC patients that were dead or alive using a two-tailed t-test. For Kaplan-Meier survival analysis, we used the Kaplan-Meier plotter tool (4) for correlation of TRIM38 mRNA expression levels with overall survival (OS) in $n=404$ patients with basal subtype breast cancer.

\section{Results}

We performed comparative transcriptome analysis using published microarray data (1) to understand in an unbiased fashion the most significant gene expression differences in the primary tumors of patients with triple negative breast cancer based on survival outcomes.

TRIM38 is differentially expressed in triple negative breast cancer and positively correlates with survival.

When comparing primary tumors of 87 patients with triple negative breast cancer, alive at time of data collection and 123 TNBC patients, dead at time of data collection, we identified the E3 ubiquitin ligase tripartite motif containing 38, TRIM38, as among the most significant transcriptional differences based on survival in human triple negative breast cancer. When ranking each of the transcripts whose expression was measured by microarray based on significance of difference in expression between the tumors of patients dead and alive, TRIM38 ranked 209 out of 53617 total transcripts, equivalent to $99.6 \%$ differential expression (Chart 1). TRIM38 differential expression in the tumors of patients based on survival outcomes was statistically significant (Figure $1 ; p=0.00516241$ ). 
TRIM38 is expressed at significantly higher levels in the breast tumors of TNBC patients when comparing based on survival.

We obtained exact mRNA expression levels for TRIM38 from breast tumors of TNBC patients dead and alive to understand the magnitude and direction of TRIM38 expression change based on survival outcomes. TRIM38 was expressed at higher levels in tumors of TNBC patients alive as compared to that of TNBC patients dead (Figure 1). Increased expression of TRIM38 in primary breast tumors was statistically significant (Figure 1: $p=0.0040$ ). TRIM38 was expressed at $-0.18 \pm 0.89$ arbitrary units (AU) in the primary tumors of TNBC patients alive, while it was expressed at $0.21 \pm 1.05 \mathrm{AU}$ in the primary tumors of TNBC patients dead.

\section{TRIM38 expression correlates with overall survival in basal subtype human breast cancer.}

We referenced a bioinformatics tool containing Kaplan-Meier survival data of human breast cancer patients to understand more thoroughly whether a relationship existed between TRIM38 primary tumor expression and patient survival in human breast cancer. We found that TRIM38 expression was positively and significantly correlated with overall survival in patients with basal or basal-like human breast cancer, a breast cancer molecular subtype which shares significant overlap with triple negative breast cancer (Figure 2; $\log$ rank $p$-value: 0.0028 for overall survival, hazard ratio: $0.56(0.38-0.82)$ (Fig. 2)). TRIM38 mRNA levels were a positive prognostic indicator in basal subtype breast cancer patients. Median OS was 39.5 months for basal subtype patients with low tumor expression of TRIM38 as compared to 115 months for basal subtype patients with high tumor expression of TRIM38.

Thus, we found through unbiased comparative transcriptome analysis that TRIM38 was among the genes whose expression was most significantly different in the primary tumors of triple negative breast cancer patients alive when compared to that of TNBC patients dead, that TRIM38 was expressed at significantly higher levels in the primary tumors of TNBC patients alive as compared to TNBC patients dead, and that TRIM38 expression was positively correlated with overall survival in the basal or basal-like molecular subtype.

\section{Discussion}

It is not understood why women of African descent (black women) are diagnosed with triple negative breast cancer more frequently than white women, and why this disease is more aggressive in black women. TRIM38 may be of relevance as a biomarker in identifying patients who are more likely to survive, perhaps as part of a panel of genes used for prognostic stratification, and the molecule itself may be important to the biology of triple negative breast cancer etiology or more likely its progression. TRIM38, in the context of viral infection, can function to stabilize cGAS and STING by sumoylation of both of these molecules which is antagonized by SENP2 $(5,6)$. Blind systems-level analyses, like this, can help shed light on the fundamental molecular characteristics of a disease whose racial propensities demand further explanation. 


\section{References}

1. Purrington, K.S., Knight III, J., Dyson, G., Ali-Fehmi, R., Schwartz, A.G., Boerner, J.L. and Bandyopadhyay, S., 2020. CLCA2 expression is associated with survival among African American women with triple negative breast cancer. PloS one, 15(4), p.e0231712.

2. Foulkes, W.D., Smith, I.E. and Reis-Filho, J.S., 2010. Triple-negative breast cancer. New England journal of medicine, 363(20), pp.1938-1948.

3. Dietze, E.C., Sistrunk, C., Miranda-Carboni, G., O'regan, R. and Seewaldt, V.L., 2015. Triple-negative breast cancer in African-American women: disparities versus biology. Nature Reviews Cancer, 15(4), pp.248-254.

4. Györffy, B., Lanczky, A., Eklund, A.C., Denkert, C., Budczies, J., Li, Q. and Szallasi, Z., 2010. An online survival analysis tool to rapidly assess the effect of 22,277 genes on breast cancer prognosis using microarray data of 1,809 patients. Breast cancer research and treatment, 123(3), pp.725-731.

5. Hu, M.M., Yang, Q., Xie, X.Q., Liao, C.Y., Lin, H., Liu, T.T., Yin, L. and Shu, H.B., 2016. Sumoylation promotes the stability of the DNA sensor cGAS and the adaptor STING to regulate the kinetics of response to DNA virus. Immunity, 45(3), pp.555-569.

6. Hu, M.M. and Shu, H.B., 2017. Multifaceted roles of TRIM38 in innate immune and inflammatory responses. Cellular \& molecular immunology, 14(4), pp.331-338. 


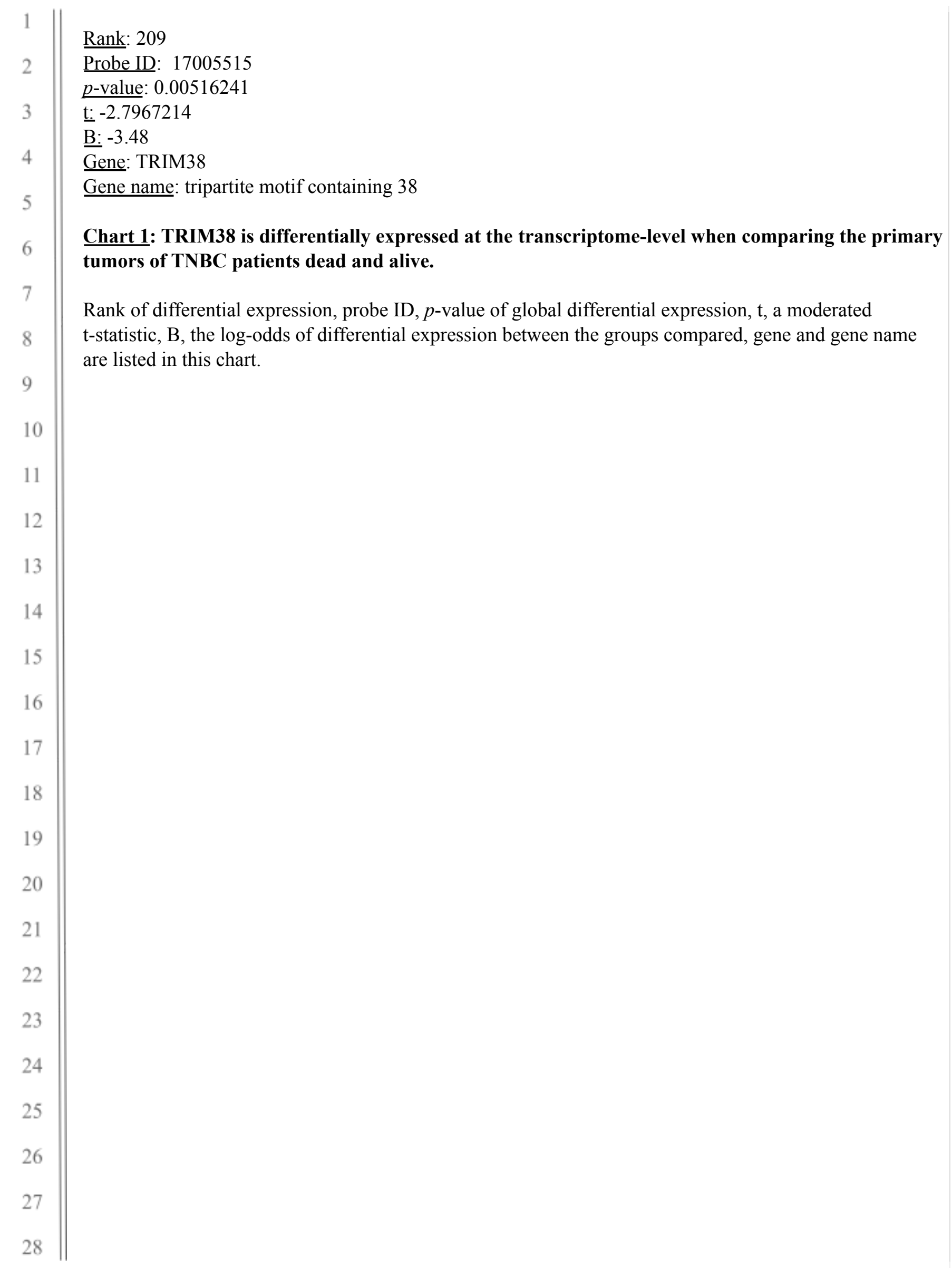


TRIM38

Figure 1: TRIM38 is expressed at significantly higher levels in the primary tumors of TNBC patients alive as compared to the primary tumors of TNBC patients dead.

The mRNA expression level of TRIM38 in the primary tumors of TNBC patients dead (left) and in primary tumors of TNBC patients alive (right) is graphically depicted with the result of a statistical test evaluating significance of difference in TRIM38 expression between the primary tumors of TNBC patients dead and alive, a $p$-value, is $p=0.0040$.

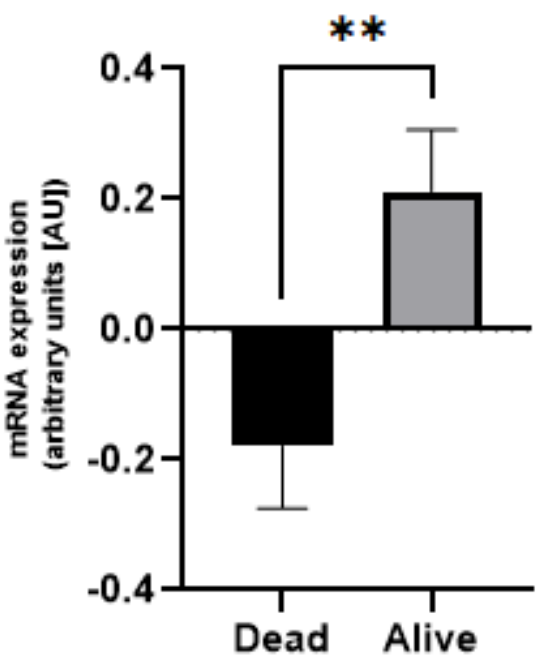




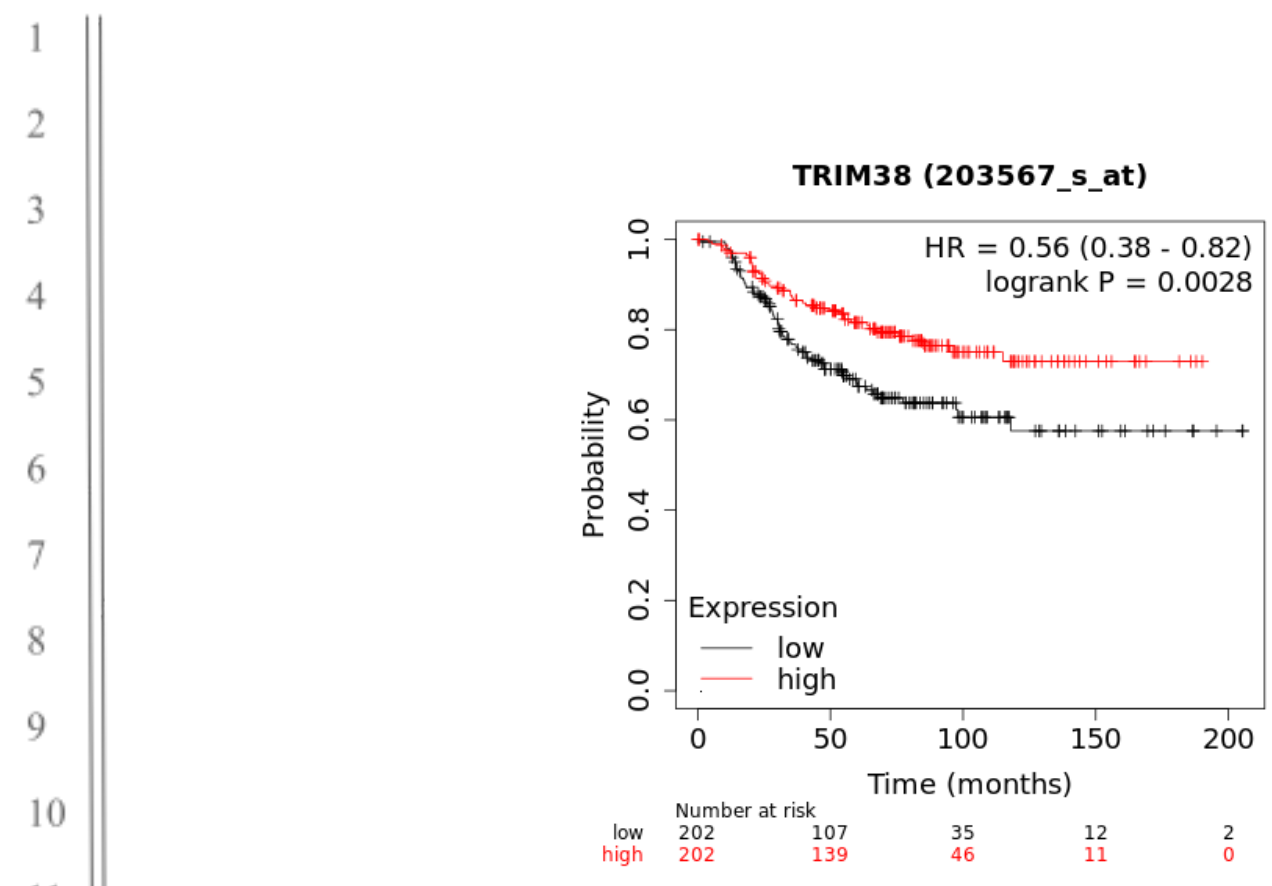

Figure 2: TRIM38 expression correlates with overall survival in basal subtype human breast cancer.

Depicted in this Kaplan-Meier plot is the probability of overall survival (OS) for $n=404$ breast cancer patients of basal subtype, based on low or high expression of TRIM38 in patient primary tumors. The log rank p-value denoting statistical significance of difference in recurrence-free survival when comparing the two groups, as well as hazard ratio for this comparison is listed above. Listed below is the number of patients at risk (number of patients alive) per interval, after stratification based on TRIM38 expression; in the first interval, number at risk is number of patients alive; in each subsequent interval, number at risk is the number at risk less those who have expired or are censored. 